\title{
Lyophilized wafers comprising carrageenan and pluronic acid for buccal drug delivery using model soluble and insoluble drugs
}

Farnoosh Kianfar, Milan Antonijevic, Babur Chowdhry, Joshua. S. Boateng*

Department of Pharmaceutical, Chemical \& Environmental Sciences, School of Science, University of Greenwich, Medway Campus, Central Avenue, Chatham Maritime, Kent ME4 $4 T B, U K$

*Corresponding author: J.S.Boateng@gre.ac.uk(Dr Joshua Boateng).

Tel: + 44 (0) 208331 8980; Fax: +44(0) 2083319805 


\begin{abstract}
Lyophilized muco-adhesive wafers with optimum drug loading for potential buccal delivery have been developed. A freeze-annealing cycle was used to obtain optimized wafers from aqueous gels containing 2\% $\kappa$-carrageenan (CAR 911), 4\% pluronic acid (F127), 4.4\% w/w polyethylene glycol with $1.8 \% \mathrm{w} / \mathrm{w}$ paracetamol or $0.8 \% \mathrm{w} / \mathrm{w}$ ibuprofen. Thermogravimetric analysis showed acceptable water content between $0.9-1.5 \%$. Differential scanning calorimetry and X-ray diffraction showed amorphous conversion for both drugs. Texture analysis showed ideal mechanical and mucoadhesion characteristics whilst both drugs remained stable over six months and drug dissolution at a salivary $\mathrm{pH}$ showed gradual release within two hours. The results show the potential of CAR 911 and F127 based wafers for buccal mucosa drug delivery.
\end{abstract}

Keywords: Amorphous, Buccal drug delivery system, Carrageenan, Dissolution characteristics, Lyophilized wafer, (Muco)-adhesion, 


\subsection{Introduction}

Mucosal drug delivery systems have many advantages such as bypassing first pass liver metabolism and avoiding GI enzymatic degradation. Drug absorption via the buccal mucosa surface and into the systemic circulation is relatively fast (compared to the skin) owing to the highly vascularized structure underneath the mucosal tissues $[1,2]$.

Lyophilisation has been used in pharmaceutical industries to improve the stability of formulations. The investigation of fundamental physical phenomena occurring during each step of freeze drying has enabled formulators to produce stable and well-designed freezedried pharmaceutical dosage forms [3]. Freeze dried products do not necessarily need to be refrigerated and can be stored at ambient temperatures. This will be favourable for labile drugs including vaccines and proteins [4].

Lyophilisation comprises a freezing step where ice crystals form and the solute becomes extremely concentrated. As the temperature falls below the glass transition temperature $\left(\mathrm{T}_{\mathrm{g}}\right)$, the system is changed into a viscous glass. Incomplete crystallization may, however, lead to sample collapse or formation of mixtures of different polymorphic forms that causes problems in characterization and manufacturing reproducibility. Frozen products can be categorized as either crystalline or amorphous glass in structure. Crystalline products have a distinct "eutectic" freezing/melting point which is referred to as the collapse temperature. Amorphous products have a corresponding "glass transition" temperature and they are much more difficult to freeze-dry. Even though most materials that are freeze dried are actually amorphous, the term "eutectic" is often used to define the freezing/melting point of sample [4].

To optimise the process of solute crystallization during freezing, thermal treatment, or "annealing", will be advantageous. Annealing is the process of heating a sample above its 
glass transition temperature (but below the eutectic and/or ice melting temperature) and allowing the glass to relax and crystallize. Metastable glass formation is possible depending on the freezing rate and/or the excipients in the formulation which should be avoided as the metastable glass will eventually crystallize $[4,5]$. Elevation of temperature above the $\mathrm{T}_{\mathrm{g}}$ during annealing reduces the viscosity and increases mobility of the solute molecules and helps prevent crystallization. However, the temperature should remain below the eutectic/collapse temperature to avoid ice melting. The thermal treatment causes an increase in the size of both solute and ice crystals resulting in reduced product resistance, faster water vapour transport and shorter primary drying times [6].

Lyophilized wafers can be prepared by freeze-drying aqueous gels of polymer(s) to form a porous polymeric inter-connecting network [7]. Swelling release systems formulated with hydrophilic polymers with several hydrogen bonding sites can be produced [8]. During drug dissolution, swelling and diffusion compete, with swelling dominating initially, by increasing the gel layer thickness and subsequently reaches a plateau due to synchronization of swelling, drug diffusion and dissolution [9] [10]. To obtain optimum bioavailability, swelling, mucoadhesion and dissolution characteristics must be optimized as they affect residence time and eventual drug release [11].

In the present study, the development and characterization of lyophilized wafers prepared by freeze-drying gels comprising the natural polysaccharide polymer $\kappa$-carrageenan (א-CAR 911), pluronic acid (F127) and polyethylene glycol 600 (PEG 600), loaded with model soluble (paracetamol - PM) and insoluble (ibuprofen - IBU) drugs for buccal delivery purposes are reported. 


\subsection{Experimental}

\subsection{Materials}

Gelcarin [(к-CAR 911, batch number: 50102070)] were gifts from BASF and obtained from the UK distributor Honeywill \& Stein LTD (Surrey, UK). Pluronic acid [F127 (batch number: 038k0071)], paracetamol [PM (batch number: RB16652)], polyethylene glycol [PEG 600 (batch number: 0001409391)] and ibuprofen [IBU (batch number: 026H1368)] were purchased from Sigma-Aldrich (Gillingham, UK).

\subsection{Gel preparation}

Different formulations were prepared based on varying concentrations of model drugs and plasticizer (PEG 600) as summarised in Table 1. Briefly, gels [12] were prepared by initially dissolving F127 in cold water $\left(<15^{\circ} \mathrm{C}\right)$ kept for two hours before addition of $\kappa$-CAR 911 and PEG 600 to the resulting solution and left overnight at room temperature to ensure complete hydration of $\kappa-\mathrm{CAR} 911$. The mixture was then heated with continuous stirring using an overhead stirrer at $40-50^{\circ} \mathrm{C}$. The model drug (PM or IBU) was added and mixing continued for a further 5-10 min. Addition of PM to the system was straight forward, however, two different strategies were employed to incorporate the maximum amount of IBU.

a. IBU (0.3-1.0\% w/w) was added to the F127 solution at the same time as א-CAR 911 and PEG 600 and left for 24 hours.

b. IBU $(0.3-1.0 \% \mathrm{w} / \mathrm{w})$ was dissolved separately in $2 \mathrm{ml}$ of ethanol and the resulting solution added to the final gel comprising к-CAR 911, F127 and PEG 600. 


\subsection{Thermal annealing (DSC studies) of gels}

DSC analysis of the gels was conducted with a Q2000 instrument (TA Instruments, Crawley, UK) under a dry nitrogen atmosphere. The instrument was calibrated with indium and sapphire prior to sample analysis. The experiment was performed by loading 3-10 $\mathrm{mg}$ of gel into T-zero aluminium pans $(75 \mu \mathrm{L})$, hermetically sealed and cooled to $-80^{\circ} \mathrm{C}$ to ensure complete freezing of all components before being heated from $-80^{\circ} \mathrm{C}$ to $80^{\circ} \mathrm{C}$ at a rate of $10^{\circ} \mathrm{C} / \mathrm{min}$.

\subsection{Freeze drying process}

An AdVantage freeze dryer (SP scientific, Ipswich, UK) was employed in automatic mode. Preliminary investigations involved obtaining wafers using an annealing procedure [13] by programming the freezing and primary/ secondary drying cycles according to preliminary experiments. $10 \mathrm{~g}$ of the gel was poured into six well polystyrene plates $(35.4$ $\mathrm{mm}$ diameter) to a height of $1 \mathrm{~cm}$. Gels were gradually cooled from room temperature to $5^{\circ} \mathrm{C}$, cooled to $0^{\circ} \mathrm{C}$ and maintained for 40 minutes, and cooled finally to $-55^{\circ} \mathrm{C}$ and maintained for 1 hour. Subsequently, annealing was performed by increasing the temperature to $-35^{\circ} \mathrm{C}$. The annealing process lasted 3 hours to provide sufficient time for large ice crystal formation. The temperature was returned to the initial temperature and maintained for 2 hours before primary drying was initiated. During primary drying, the temperature was increased from $-55^{\circ} \mathrm{C}$ to $10^{\circ} \mathrm{C}$ and eventually $0^{\circ} \mathrm{C}\left(5^{\circ} \mathrm{C}\right.$ lower than the eutectic points) to prevent melt back and preserve the stability of all the components. The whole primary drying procedure was 8 hours at $-10^{\circ} \mathrm{C}$ followed by 6 hours at $-0^{\circ} \mathrm{C}$. To increase the stability of the freeze-dried wafers at room temperature, secondary drying was performed for 2 hours to further reduce residual water content. 


\subsection{Thermal Analysis}

(i) Thermogravimetric analysis (TGA)

TGA was used to determine the residual water in the wafers and the effect of concentration of model drugs on their water content. About 3-10 mg of sample was accurately weighed, placed in aluminium pans $(100 \mu \mathrm{L})$ and weight loss measured using a high resolution TGA 2950 instrument (TA Instruments, Crawley- UK). The experimental program involved heating the samples from $25^{\circ} \mathrm{C}$ to $150^{\circ} \mathrm{C}$ at a heating rate of $10^{\circ} \mathrm{C} / \mathrm{min}$. Each experiment was performed three times.

(ii) Differential scanning calorimetry (DSC)

The thermal profiles of the optimized wafers containing IBU or PM were investigated. Samples were placed in T-zero pans, cooled down to $-80^{\circ} \mathrm{C}$, maintained at this temperature for 5 minutes before heating up to $180^{\circ} \mathrm{C}$ at a rate of $10^{\circ} \mathrm{C} / \mathrm{min}$ and kept at this temperature for 3 minutes to allow complete melting. The run proceeded by cooling the sample at a rate of $-10^{\circ} \mathrm{C} / \mathrm{min}$ back to $-80^{\circ} \mathrm{C}$. This process was repeated twice to investigate the stability of the polymer matrix and model drugs during the heating cycle.

\subsection{X-Ray powder diffraction (XRPD)}

XRPD was used to determine the physical form of the individual components present in the wafer (polymers and drugs). A D8 Advance XRPD diffractometer (Bruker, Coventry, UK) equipped with a Lyn $\mathrm{X}$ - Iris detector and $6.5 \mathrm{~mm}$ slit size was employed to obtain results in reflection and transmission modes. The instrument was set at $40 \mathrm{kV}$ and $40 \mathrm{~mA}$ 
with primary solar slit of $4^{\circ}$ and a secondary solar slit of $2.5 \mathrm{~mm}$ while the scattered slit was $0.6 \mathrm{~mm}$. Samples were scanned at a speed of $0.02^{\circ}, 2 \theta$ step size every 0.1 seconds.

\subsection{HPLC analysis}

HPLC analysis was performed using an ODS C18 reverse phase $(5 \mu \mathrm{m}$ particle size) column (Hichrom H50DS-3814), mobile phases of methanol: water: ortho-phosphoric acid (74:24:2), flow rate $1.50 \mathrm{ml} / \mathrm{min}$ for IBU and methanol: water: ortho-phosphoric acid $(25: 75: 3 \mathrm{v} / \mathrm{v} \%)$, flow rate $1 \mathrm{~mL} / \mathrm{min}$ for PM and diode array UV detection at 214 and $245 \mathrm{~nm}$ for IBU and PM respectively. A $0.50 \mathrm{mg} / \mathrm{ml}$ standard solution of IBU was prepared, serially diluted $(0.05,0.075,0.10,0.125$ and $0.15 \mathrm{mg} / \mathrm{ml})$ used to plot a calibration curve. The calibration curve for PM was plotted by preparing $1 \mathrm{mg} / \mathrm{ml}$ standard solution of PM and serially diluted to $0.10,0.15,0.20,0.25,0.30 \mathrm{mg} / \mathrm{ml}$.

\subsection{Stability test}

The formulations wrapped in paraffin film (to prevent moisture absorption by $\kappa-C A R$ 911, which is hygroscopic) were stored at room temperature and $45 \%$ relative humidity (RH) over a six month period and the drug content assayed monthly using HPLC. Drug stability over the storage period was analysed by dissolving wafers in deionized water prior to analysing on the HPLC. Samples were analysed at the following time points: day 1 (freshly prepared), 7 days, and then at 2, 4 and 6 months. Pure crystalline IBU and PM powders stored under similar conditions as the wafers were used as controls. 


\subsection{Scanning electron microscopy (SEM)}

SEM was used to evaluate the topographic characteristics and morphology of the wafers. The analyses were carried out using a Jeol Instrument (Japan) with back scattered electrons and artificial shadowing ability with uncoated samples at low vacuum $(<20 \mathrm{~Pa})$ and an accelerating voltage of $20 \mathrm{kV}$.

\subsection{Texture analysis}

(i) Mechanical characteristics

This was used to select optimized wafers with acceptable flexibility (PEG 600 concentration) for drug loading as well as to determine the effect of increasing drug content. The instrument employed was a "Texture analyser HD-plus" with Exponent software to plot and display the data. Before compression, the thickness of the wafers containing 4.4-5.5\% w/w PEG 600 was measured by a micro screw-gauge (four edges and one in the middle). The average thickness (ranging from 3.5-3.7 $\mathrm{mm}$ ) of each specific sample was entered into the texture analyser software prior to compression. The texture analyser was set to compression mode and a force was applied by a P6 (6 mm diameter) cylindrical stainless steel probe at a speed of $0.1 \mathrm{~mm} / \mathrm{sec}$ to compress the wafer to the depth of $2 \mathrm{~mm}$, held for $2 \mathrm{sec}$ before returning to the starting position at a speed of $1 \mathrm{~mm} / \mathrm{sec}$. The peak compression force $(\mathrm{CF})$ representing resistance to compression and work of compression (WOC) of the wafers containing different concentrations of PEG 600 and model drugs were measured.

\section{(ii) In vitro mucoadhesivity studies}

Mucoadhesivity experiments were conducted by employing a $75 \mathrm{~mm}$ diameter stainless steel probe attached to the Texture analyser-HD Plus instrument. The lyophilised 
wafer's specimen was cut to $3 \mathrm{~cm} \times 3 \mathrm{~cm}$ size and attached to the probe using double sided adhesive tape. A Petri dish containing set agar gel, equilibrated with $200 \mu \mathrm{L}$ buffer solution $(\mathrm{pH}=6.2)$ was used as the mucosal substrate to simulate the buccal mucosa. The sample was placed in contact with the agar surface, maintained for one minute to allow hydration and maximum contact between the sample and the mucosal substrate. The Texture Analyser was programmed to work in tension mode and the probe detached at a pre-test speed of 0.5 $\mathrm{mm} / \mathrm{sec}$, and test speed of $1 \mathrm{~mm} / \mathrm{sec}$. The maximum force applied to separate the specimen from the agar surface and work of adhesion (WOA), were determined.

\subsection{Hydration (swelling) studies}

These studies investigated the maximum time required for the wafers to completely hydrate and their maximum swelling capacity in phosphate buffer solution. The buffer was prepared by the addition of $100 \mathrm{ml}$ of $\mathrm{KH} 2 \mathrm{SO} 4(0.1 \mathrm{M})$ to $13 \mathrm{ml}$ of $\mathrm{NaOH}(0.1 \mathrm{M})$. Since the secretion of saliva is $0.3 \mathrm{ml} / \mathrm{min}$ [14], the volume of the medium was $42 \mathrm{ml}$ and wafers were immersed for 140 minutes. Wafers were cut to $3 \mathrm{~cm} \times 3 \mathrm{~cm}$ square strips, weighed and placed in the buffer solution $(\mathrm{pH}=6.2)$. The weight changes in the sample were measured every 20 minutes till constant weight up to a maximum of 140 minutes and the data plotted as weight change versus time. Calculation of $\%$ swelling (\% weight change) was determined using equation 1 where $W_{0}$ and $W_{t}$ are the weights of the lyophilized wafer initially and at time $\mathrm{t}$ respectively. Each data point represents the mean $( \pm$ s.d.), of three replicates. $\%$ Swelling $=\frac{W_{0}-W_{t}}{W_{0}} \times 100$ 


\subsection{Drug dissolution studies}

Drug dissolution studies were performed at $37^{\circ} \mathrm{C}$ using $42 \mathrm{ml}$ buffer solution (as in swelling studies) with $\mathrm{pH}=6.2$ simulating that of saliva. Dissolution media was sampled at intervals of 2 minutes from time zero and drug measured using the HPLC method above. Drug released $(\mathrm{mg})$ were calculated from the calibration curves and cumulative percentage release versus time profiles plotted. The kinetics of PM and IBU release from the wafer was assessed by fitting the dissolution data (percentage cumulative release against time) to the Higuchi, Hixon-Crowell, Korsmeyer-Peppas, first or zero order equations in order to determine the drug release mechanism.

\subsection{Statistical analysis}

To compare the dissolution and mucoadhesion results statistical analyses were employed by one-way ANOVA. The results were considered statistically significant if a p value of $<0.05$ was obtained. All measurements were performed in triplicate and the results are presented as the mean \pm standard deviation (s.d).

\subsection{Results and discussion}

\subsection{Preliminary development}

Establishing a suitable thermal profile for any gel prior to freeze-drying was critical in developing an optimized lyophilization cycle. Because of possible changes to wafer properties due to addition of the model drug or changes in plasticizer concentration, complimentary DSC data was required to detect the critical points. The eutectic point $T_{c}\left(T_{e u}\right)$ was observed at $7.91{ }^{\circ} \mathrm{C}$ and $10.91^{\circ} \mathrm{C}$ for IBU and PM loaded wafers respectively. To ensure 
that the formulation did not collapse from melt back and/or incomplete ice removal the product temperature was maintained below the $T_{c} / T_{\text {eu }}$ (collapse/eutectic temperature) to retain interstitial space in the solid phase and make it capable of supporting its own weight after the ice removal and subsequently preserve the wafer's structure $[15,16]$.

Visual evaluation of wafers

Evaluation of the wafers formulated with 2\% к-CAR 911, 4\% F127 and varying concentrations of PEG 600 with the freeze annealing process were conducted and optimum wafers were selected based on the following criteria:

- Softness: wafers must be soft and easy to apply on the mucosal tissues.

- Plasticity: the wafer should not be brittle and fragile as it will affect physical and mechanical stability during handling as well as potential contact irritation during application.

- Thickness: Ideal wafer must have optimum thickness (less than $1 \mathrm{~cm}$ pre-hydration) to ensure convenience for patients when applied to the buccal mucosa area. Thick wafers present the possibility of accidental chewing and dislodging by tongue movement. In addition, thickness of the wafer affects the drug release rate since it determines the diffusion distance through the initial gel formed following hydration.

Based on visual evaluations gels comprising 2\% w/w $\kappa-C A R ~ 911,4 \%$ w/w F127 and $4.4 \% \mathrm{w} / \mathrm{w}$ PEG 600 with maximum drug loading of $1.8 \% \mathrm{w} / \mathrm{w}$ PM or $0.8 \% \mathrm{w} / \mathrm{w}$ IBU was selected as optimum for freeze-drying. 


\subsection{Thermal analysis studies}

(i) Thermogravimetric analysis (TGA)

The residual water determined immediately after freeze-drying for wafers prepared

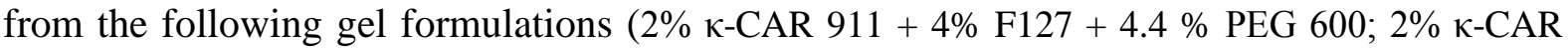
$911+4 \%$ F $127+4.4 \%$ PEG $600+0.8 \%$ IBU and 2\% $\%$-CAR $911+4 \%$ F127 + $4.4 \%$ PEG $600+$ $1.8 \%$ PM) were respectively $1.5( \pm 0.1), 1.1( \pm 0.2)$ and $0.9( \pm 0.01)$. Following freeze-drying, the water content should be at a minimum level, typically between $0.5 \%$ and $3 \%$ [18]. This is because there is a potential for high residual water to act as seeds to initiate and accelerate the crystallization of amorphous drug during storage. Water is also a very effective plasticizer which significantly depresses the $\mathrm{T}_{\mathrm{g}}$ of the amorphous drug and excipients by increasing molecular mobility which can result in instability [19]. The results confirmed that annealing is a desirable process to develop wafers with appropriate stability due to lower residual water content [13].

The water content of drug (IBU and PM) loaded wafers showed that addition of model drugs resulted in a reduction of residual water in the wafer. This can be attributed to the competition of the drug with water molecules for interaction with $\kappa-C A R 911$. Interestingly, the results showed that the amount of residual water was reduced considerably due to addition of PM compared with IBU loaded wafers .Wu and McGinity [20] have shown that $\mathrm{T}_{\mathrm{g}}$ of polymers would be decreased due to the presence of IBU within the polymeric wafer. 
(ii) Differential scanning calorimetry (DSC)

The DSC results for the blank and drug loaded wafers demonstrated that both model drugs were present in amorphous form. Figure $1 \mathrm{a} \& \mathrm{~b}$ represent DSC thermograms of pure IBU which showed that during the first heating cycle a sharp melting transition was detected at $77.68^{\circ} \mathrm{C}$ and following the rapid cooling, glass transition of the amorphous form was detected at $-45.08^{\circ} \mathrm{C}$ though this transition was shifted to $-59.08^{\circ} \mathrm{C}$ within the wafer. Figure 1c also showed three sharp peaks $\left(-1.78^{\circ} \mathrm{C}, 38.6^{\circ} \mathrm{C}\right.$ and $\left.26.46^{\circ} \mathrm{C}\right)$ corresponding to PEG 600 , F127 and the mixture of these two polymers [21] respectively and the disappearance of the IBU melting transition.

Similarly, pure PM (Figure 1d\&e) showed a sharp melting peak at $169.76^{\circ} \mathrm{C}$ in the first heating cycle. During the rapid cooling, the crystalline drug was changed to amorphous form. However, the amorphous form is unstable and recrystallized at $80.53^{\circ} \mathrm{C}$. The $\mathrm{T}_{\mathrm{g}}$ of $\mathrm{PM}$ was detected at $24.11^{\circ} \mathrm{C}$ and a sharp melting peak confirmed the recrystallization process. This result was compared with thermogram of the PM loaded wafer (Figure 1f) and showed a stable amorphous form of PM in the wafer with glass transition at $21.77^{\circ} \mathrm{C}$ with the absence of the expected melt peaks $158.15^{\circ} \mathrm{C}$ (orthorhombic polymorph) and $169.76^{\circ} \mathrm{C}$ (monoclinic), confirming the existence of amorphous form of the drug in the wafer.

An interesting finding was the absence of the melting transition of the mixture of PEG 600/F127 in PM loaded wafers possibly due to less water content compared with IBU loaded wafer. As noted above, the PM molecules compete with water molecules for interaction with binding sites on $\kappa$-CAR 911 through hydrogen bonds. This is possible given the higher amounts of PM within the polymeric matrix compared with IBU loaded wafers where the lower water content limited the interaction of PEG 600 and F127 as the presence of water in the system has a significant effect on interaction between PEG 600 and F127 [21]. 


\section{$3.3 X$-ray powder diffraction $(X R P D)$}

Figures $2 \mathrm{a} \& \mathrm{~b}$ show the XRPD patterns for wafers containing IBU and PM. The X-ray patterns shown in Figure 2a correspond to the wafer containing all components ( $\kappa$-CAR 911, F127, PEG 600 and IBU) which represents all the crystalline molecules present within the wafer's matrix. The results demonstrate the absence of the main peak of crystalline IBU that should have appeared at $16.2(2 \theta)$ according to the XRPD library data base. Similar results were observed for PM loaded wafer (Figure 2b), as evidenced by the absence of the main crystalline peak expected at about 24.5 (20) for PM. This confirmed the DSC results and showed that the crystalline IBU or PM originally added to the system was transformed into amorphous form during freeze-drying to produce the wafers.

\subsection{Stability test}

HPLC assay showed that the amount of drug within the wafers during six months storage at room temperature and $45 \pm 5 \% \mathrm{RH}$ remained fairly constant. For IBU wafers, the percent drug content varied from $99.86 \%$ at time zero to $99.28 \%$ after six months of storage whilst for PM loaded wafers, the percent drug content varied from $99.90 \%$ at time zero to 99.15\% after six months. These results show the ability of $\kappa$-CAR 911, F127 and PEG 600 based wafers to maintain the chemical stability of IBU and PM. However, this was over six months storage and the stability profiles over a longer storage period corresponding to the expected shelf time as well as under accelerated conditions will need to be investigated.

\subsection{Scanning electron microscopy (SEM)}

SEM was employed to assess the surface characteristics (morphology) of the lyophilized wafers and determine its relation to swelling, dissolution and mucoadhesion 
characteristics. The SEM images of different formulations are shown in Figure 3a-3d with different degrees of porosity. The SEM images of drug loaded wafers showed significant

differences in surface topography of wafers formulated with the two model drugs. The PM loaded wafer at high drug loading $(1.8 \% \mathrm{w} / \mathrm{w})$ showed the least porosity as the surface texture appeared to be leafy while the IBU loaded wafer showed a more porous texture with uniform pore size distribution. The leafy surface structure and reduced porosity could be attributed to the higher amounts of PM incorporated in the wafer's matrix. However, the wafers containing lower amounts $(0.8 \% \mathrm{w} / \mathrm{w})$ of PM (Figure $3 \mathrm{~d})$ showed a more porous structure. Wafers with larger pores allow a more rapid ingress of water during dissolution which is expected to increase drug release rates.

\subsection{Texture analysis}

\section{(i) Mechanical characteristics}

Figure 4a shows that addition of IBU increased the work of compression (WOC) [17] while PM decreased it to approximately half that of IBU loaded wafers, and also lower than the blank (non drug loaded wafer). These results combined with SEM observations showed that addition of PM decreased mechanical strength due possibly to the reduced availability of free polymer as a result of the higher amounts of PM distributed throughout the matrix.

To evaluate the effect of PEG 600 concentration, a new set of experiments were conducted. Optimised wafers prepared from gels containing 2\% к-CAR 911, 4\% F127 and $1.8 \%$ PM with varying PEG 600 concentrations (4.4, 5.0 and 5.5\%) showed WOC (Nmm) values of $3.4( \pm 0.3), 3.5( \pm 0.5)$ and $2.9 \pm 0.2$ respectively. The peak compression force values $(\mathrm{N})$ were also respectively $4.5( \pm 0.2), 3.4( \pm 0.3)$ and $3.1( \pm 0.2)$. The results showed 
that increasing the levels of PEG 600 beyond $4.4 \% \mathrm{w} / \mathrm{w}$ did show further increase in WOC, therefore PEG 600 at 4.4\% w/w within the gel for both blank and drug loaded wafers was the concentration of choice.

\section{(ii) In vitro mucoadhesion}

During the mucoadhesivity studies three parameters were measured; (i) WOA (work required to overcome the attractive forces between the surface of the wafer and the agar), (ii) stickiness factor (maximum force required for detaching the wafer from the surface of the agar) and (iii) cohesiveness (the distance the wafer travels in $\mathrm{mm}$ to detach from the agar surface). All of these factors are correlated with the strength of the bonds formed between the polymeric matrix and agar during the contact time [30].

Figure $4 \mathrm{~b}$ shows the WOA and stickiness for the various formulations. Both of the above parameters were significantly affected by PEG 600. The results showed that maximum mucoadhesivity was observed for the wafer comprising $2 \% \mathrm{w} / \mathrm{w}$ of $\kappa-\mathrm{CAR} 911$ and $4 \% \mathrm{w} / \mathrm{w}$ F127 in the presence of 4.4\% PEG 600 which previously showed appropriate flexibility and remained stable during handling. Shaikh and co workers [26] reported that the presence of PEG 600 increased the quantity of residual water that resulted in drug precipitation and a slight decrease in the adhesive performance. Therefore, the amount of the PEG 600 in the system should be kept at the lowest optimum level. Further, incorporating the hydrophobic drug (IBU) in the wafers' matrix resulted in a slight decrease in mucoadhesion compared with blank wafer though the difference was not significant $(\mathrm{p}=0.0532)$. Interestingly, addition of PM which is a hydrophilic drug resulted in an increase in the WOA, stickiness and cohesiveness. 
к-CAR 911 exhibit bioadhesive characteristics effect due to its anionic nature and numerous hydrogen bonding groups which allow it to bind to mucosal tissues more efficiently. Scientifically, ionisable polymers existing in their unionized form are able to form non-covalent bonds with mucus glycoprotein [27]. Based on the chemical structure of mucin, there is the feasibility of hydrogen bond formation between mucin and $\kappa$-CAR 911 as well as formation of ionic bonds between the sulphate group in $\kappa$-CAR 911 and $\mathrm{NH} 2$ group in mucin which could result in a stronger mucoadhesion force. Ruiz and Ghaly [28] have previously reported the ability of CAR based tablets to adhere to agar gel surface. In addition to hydrogen bonds, mucoadhesion can be generated due to van der Waals and dative bonds or entanglement between polymeric matrix and agar [29].

\subsection{Swelling studies}

Figure 5a shows the swelling profiles of optimised wafers formulated with $2 \% \mathrm{w} / \mathrm{w}$ CAR 911, 4\% w/w F127 and 4.4\% w/w PEG 600 containing either IBU or PM. The results showed that the maximum swelling capacity for wafers in buffer solution occurred within 40 minutes. In particular, the blank and IBU loaded wafers hydrated relatively more rapidly. The drug loaded formulations attained maximum swelling of $720 \%$ and $500 \%$ for IBU and PM loaded wafers respectively within 20 minutes after which the weight changes remained constant. The hydration and swelling capacity for IBU loaded wafer in the buffer solution was higher than blank and PM loaded wafer. This phenomenon can be related to the more extensive porosity in IBU wafer and acidic nature of IBU compared to PM molecules which interacted more readily with $\kappa-\mathrm{CAR} 911$ through hydrogen bond formation. These differences impact on mucoadhesion and expected to affect drug release rates. 


\subsection{Drug dissolution studies}

Figure $5 \mathrm{~b}$ compares the dissolution profiles for IBU and PM within the wafers in buffer solution. PM is a weak basic drug with pKa 9.5 and is stable in alkali condition whilst IBU is a relatively weak acidic with the pKa about 4.4 and low solubility in water or at acid $\mathrm{pH}$. IBU was released from the wafer's matrix more slowly initially however; it showed faster release profiles reaching about $30 \%$ in 20 minutes and ultimately $75 \%$ after 120 minutes. In contrast, PM was initially released from wafer's matrix relatively fast and reached a plateau (about 40\%) within 10 min. The maximum \% drug release from the PM loaded wafer reached 50\% after 120 minutes, which was considerably lower compared with IBU loaded wafer. These differences can be explained by the swelling studies as the swelling capacity of IBU loaded wafers was significantly higher than the PM loaded wafers. The release profiles for both IBU and PM from the wafers were generally similar and seems to confirm the observation that IBU and PM were both present in the wafers in the amorphous form [31]. Conventionally, the amorphous form of a drug possessing higher water solubility is expected to have higher rates of dissolution and therefore may account for the higher than expected rates of release by diffusion and eventual erosion of the polymer matrix when compared with the crystalline drug.

The $\mathrm{R}^{2}$ values show that the kinetic model that best fit the release data for both drugs consistently was the Korsmeyer-Peppas (equation 2) which involved a combination of diffusion in the first step followed by erosion of the polymeric matrix.

$$
\ln \left(\frac{Q_{\infty}}{Q_{t}}\right)=\ln k+n \ln t
$$


Where Qt is the amount of drug released in time $t, Q_{\infty}$ is the initial amount of drug present in the film, $\mathrm{k}$ is a constant comprising the structural and geometric characteristics of the formulation and $\mathrm{n}$ is the release exponent $[22,23]$. As shown in Table 2, the $\mathrm{R}^{2}$ values determined were 0.99 and 0.96 for IBU and PM wafers respectively. Since, IBU loaded wafers showed $0.45>\mathrm{n}>0.89$, the drug release from the polymeric matrix was based on anomalous transport which means that diffusion and erosion played the key role in drug release behaviour [24]. The $\mathrm{n}$ value for PM loaded wafers however, showed the value of $\mathrm{n} \leq$ 0.45 corresponding to case I diffusional (Table 2) due to diffusion through the pores of the matrix when the polymer chains relax and is known as diffusion-controlled release systems [25].

\subsection{Conclusion}

The development and optimisation of buccal wafers has been achieved by freeze-

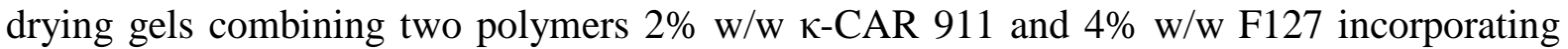
$4.4 \% \mathrm{w} / \mathrm{w}$ PEG 600 as well as $0.8 \% \mathrm{w} / \mathrm{w}$ or $1.8 \% \mathrm{w} / \mathrm{w}$ IBU and PM loaded wafers. The results also showed the conversion of crystalline drugs to the amorphous form during gel formation and freeze-drying and the wafer's matrix demonstrated the ability to maintain the two model drugs in a stable amorphous form during storage over a six month period. The wafers showed ideal release patterns in conditions simulating those of saliva and coupled with the desirable mucoadhesive characteristics, have potential for buccal drug delivery.

\section{Conflict of interest}

The authors report no conflict of interest 


\subsection{References}

1. R. P. Dixit and S. P. Puthli, J. Cont. Rel. 139 (2009) 94-107.

2. B. Luppi, F. Bigucci, A. Abruzzo, G. Corace and T. Cerchiara, V, Eur. J. Pharm. Biopharm. 75 (2010) 381-387.

3. S. C. Tsinontides, P. Rajniak, D. Pham, W.A. Hunke, J. Placek and S.D. Reynolds, Int. J. Pharm. 280 (2004) 1-16.

4. S.C. Schneid and H. Gieseler, VirTis / FTS Company, (2009), 1-4.

5. http://www.nbtc.cornell.edu/facilities/freeze_drying_guide.pdf (Accessed on 1 February 2012).

6. A. Pyne, R. Surana and R. Suryanarayanan, Pharm. Res. 19 (2002) 901-908.

7. K. H. Matthews, H. N. E. Stevens, A. D. Auffret, M. J. Humphrey and G. M. Eccleston, Int. J. Pharm. 356 (2008) 110-120.

8. J. S. Boateng, K. H. Matthews, A. D. Auffret, M. J. Humphrey, G. M. Eccleston, H. N. E. Stevens. Drug Dev. Ind. 38(1) (2012) 47-54.

9. C. Lin and A. T. Metters. Adv. Drug. Deliv. Rev. 58(12-13) (2006) 1379-1408.

10. B. Narasimhan, N. A. Peppas, J. Pharm. Sci. 86(3) (1997) 297-304.

11. N. R. Mathias, M. A. Hussein, J. Pharm. Sci. 99 (2009) 1-20.

12. F. Kianfar, B. Z. Chowdhry, M. D. Antonijevic, J. S. Boateng. UKPharmSci 2011- The Science of Medicines Conference Proceedings 31th August -3rd September, Nottingham, 2011.

13. F. Kianfar, B. Z. Chowdhry, M. A. Antonijevic and J. S. Boateng, J. AAPS. PharmSci. 13(S2) (2011) W4274.

14. L. Eliasson, D. Birkhed and A. Carlen, Arch. Oral. Bio. 54(3) (2009) 263-267.

15. www.pharmaceuticalonline.com/article.mvc/Basic-Cycle-Development-Techniques-ForLyophi-0001> [Accessed on 28 August 2011]. 
16. J. McGinnity, Freeze-dry microscopy improves pharmaceutical efficiency, cost and quality, 1st edition, The McCrone Group's College, Westmont, Illinois 2009, 48-56.

17. C. De Brabander, G. Van Den Mooter, C. Vervaet and J.P. Remon. Characterization of ibuprofen as a non-traditional plasticizer of ethyl cellulose, J. Pharm. Sci. 91 (7) (2002) $1675-1785$.

18. www.biopharma.co.uk- date accessed 20/06/2011.

19. N. Passerini and D. Q. Craig, J. Contr. Rel. 73(1) (2001) 111-115.

20. C. Wu and J. W. McGinity, AAPS. Pharm. Sci. Tech. 2(4) (2001) 1-9.

21. F. Kianfar, B. Z. Chowdhry, J. S. Boateng, M. D. Antonijevic, J. Pharm. Pharmacol. 62, (2010) 1341.

22. P. L. Ritger and N. A. Peppas, J. Contr. Rel. 5 (1987) 37-42.

23. J. S. Boateng, H. N. Stevens, G. M. Eccleston, A. D. Auffret, M. J. Humphrey and K.H. Matthews, Drug. Dev. Ind. Pharm. 35(8) (2009) 986-996.

24. N. Balji and N. S Peppas. J. Pharm. Sci. 89 (1996) 297-304.

25. J. Siepmann and N. A. Peppas. Adv. Drug. Del. Rev. 48 (2001) 139-157.

26. R. Shaikh, T. R. Raj Singh, M. J. Garland, A. D. Woolfson and R. F. Donnelly, J. Pharm. Bioallied Sci. 3 (2011) 89-100.

27. S. Roy, K. Pal, A. Anis, K. Pramanik and B. Prabhakar, Des. Monomers. Polym. 12 (2009) 483-495.

28. G. Ruiz and E. Ghaly, RLPO Vitae, 13 (2006) 1-2.

29. J. D. Smart, Expert. Opin. Drug. Deliv, 3 (2005) 507-17.

30. K. Bansal, M. K. Rawat, A. Jain, A. Rajput, T. P. Chaturvedi, S. Singh, AAPS PharmSciTech. 10(3) (2009) 716-723.

31. F. Kianfar, B. Z. Chowdhry, M. D. Antonijevic and J. S. Boateng, Drug. Dev. Ind. Pharm. 2011, DOI: 10.3109/03639045.2011.644294. 
Figure 1 DSC thermograms showing (a) melting point of pure IBU (b) Tg of pure IBU after rapid cooling from the melt (c) wafer produced from gels containing $2 \% \mathrm{w} / \mathrm{w} \kappa-\mathrm{CAR} 911+$ 4\% w/w F127+ 4.4\% w/w PEG $600+0.8 \%$ w/w IBU (d) melting point of pure PM (e) Tg of pure PM after rapid cooling from melt (f) wafer produced from gels containing $2 \% \mathrm{w} / \mathrm{W} \kappa-$ CAR $911+4 \%$ w/w F127 + 4.4\% w/w PEG $600+1.8 \%$ w/w PM.

Figure 2 XRPD diffractograms showing the crystallographic patterns of (a) wafer containing IBU (b) wafer containing PM. (Both demonstrate the absence of the peak belonging to the crystalline forms of the two model drugs and confirm the existence of amorphous PM and IBU within the wafers).

Figure 3 SEM images showing the surface morphology of (a) blank wafer (b) PM loaded wafer from gel containing $1.8 \%$ of the drug (c) IBU loaded wafer from gel containing $0.8 \%$ of drug and (d) PM loaded wafer from gel containing $0.8 \%$ of the drug.

Figure 4 (a) Work of compression (Nmm) profiles for blank or drug loaded wafers produced from gels comprising 2\% w/w $\kappa-$ CAR $911+4 \%$ w/w F127 + 4.4\% w/w PEG 600 or loaded with $0.8 \%$ IBU or $1.8 \%$ PM; (b) Mucoadhesion profiles showing work of adhesion (WOA), stickiness and cohesiveness of blank and drug (IBU or PM) loaded wafers.

Figure 5 (a) Hydration profile showing the $\%$ swelling for the blank and drug laded wafers (IBU or PM) in phosphate buffer $\{$ mean \pm s.d. $n=3\}$; (b) Drug dissolution plots showing the release profiles of drugs form the wafers loaded with IBU and PM. 
Table 1 Composition of the gels prepared during formulation development and optimization process for wafers

\begin{tabular}{cccc}
\hline$\kappa-C A R ~ 911$ & F127 & PEG 600 & Drugs \\
$(\% \mathrm{w} / \mathrm{w})$ & $(\% \mathrm{w} / \mathrm{w})$ & $(\% \mathrm{w} / \mathrm{w})$ & $(\% \mathrm{w} / \mathrm{w})$ \\
\hline 2.0 & 4.0 & 4.4 & $0.8 \mathrm{IBU}$ \\
2.0 & 4.0 & 5.0 & $0.8 \mathrm{IBU}$ \\
2.0 & 4.0 & 5.5 & $0.8 \mathrm{IBU}$ \\
2.0 & 4.0 & 4.4 & $1.8 \mathrm{PM}$ \\
2.0 & 4.0 & 5.0 & $1.8 \mathrm{PM}$ \\
2.0 & 4.0 & 5.5 & $1.8 \mathrm{PM}$ \\
2.0 & 4.0 & 4.4 & $0.9 \mathrm{IBU}$ \\
2.0 & 4.0 & 4.4 & $1.9 \mathrm{PM}$ \\
2.0 & 4.0 & 4.4 & $0.8 \mathrm{PM}$ \\
2.0 & 4.0 & 4.4 & $0.6 \mathrm{IBU}$ \\
\hline
\end{tabular}


Table 2 Fitting of dissolution data for the two model drugs to various kinetic models to determine the mechanism of drug release from the wafers.

\begin{tabular}{llll}
\hline \multicolumn{1}{c}{ Dissolution models } & IBU wafer & PM wafer \\
\hline Zero order & $\mathrm{k}_{0}\left(\% \mathrm{hr}^{-1}\right)$ & 0.95 & 1.25 \\
& $\mathrm{R}^{2}$ & 0.95 & 0.88 \\
First order & $\mathrm{RSM}$ & 159.21 & 101.59 \\
& $\mathrm{k}_{1}\left(\mathrm{~h}^{-1}\right)$ & $1.3 \times 10^{-2}$ & $8.410^{-3}$ \\
& $\mathrm{R}^{2}$ & 0.99 & 0.90 \\
Higuchi & $\mathrm{RSM}$ & 4.04 & 409.78 \\
& $\mathrm{k}_{\mathrm{H}}\left(\% \mathrm{hr}^{-1 / 2}\right)$ & 6.72 & 5.40 \\
& $\mathrm{R}^{2}$ & 0.97 & 0.00 \\
& $\mathrm{RSM}$ & 40.05 & 212.00 \\
Hixson-Crowell & $\mathrm{k}_{\mathrm{HC}}\left(\% \mathrm{~h}^{-1 / 3}\right)$ & $3.7 \times 10^{-3}$ & $2.5 \times 10^{-3}$ \\
& $\mathrm{R}^{2}$ & 0.99 & 0.80 \\
& $\mathrm{RSM}$ & 9.88 & 444.49 \\
& $\mathrm{kP}\left(\mathrm{hr}^{-\mathrm{n}}\right)$ & 3.13 & 31.59 \\
& $\mathrm{R}^{2}$ & 0.99 & 0.96 \\
& $\mathrm{n}$ & 0.68 & 0.06 \\
& $\mathrm{RSM}$ & 14.17 & 7.63 \\
\hline
\end{tabular}



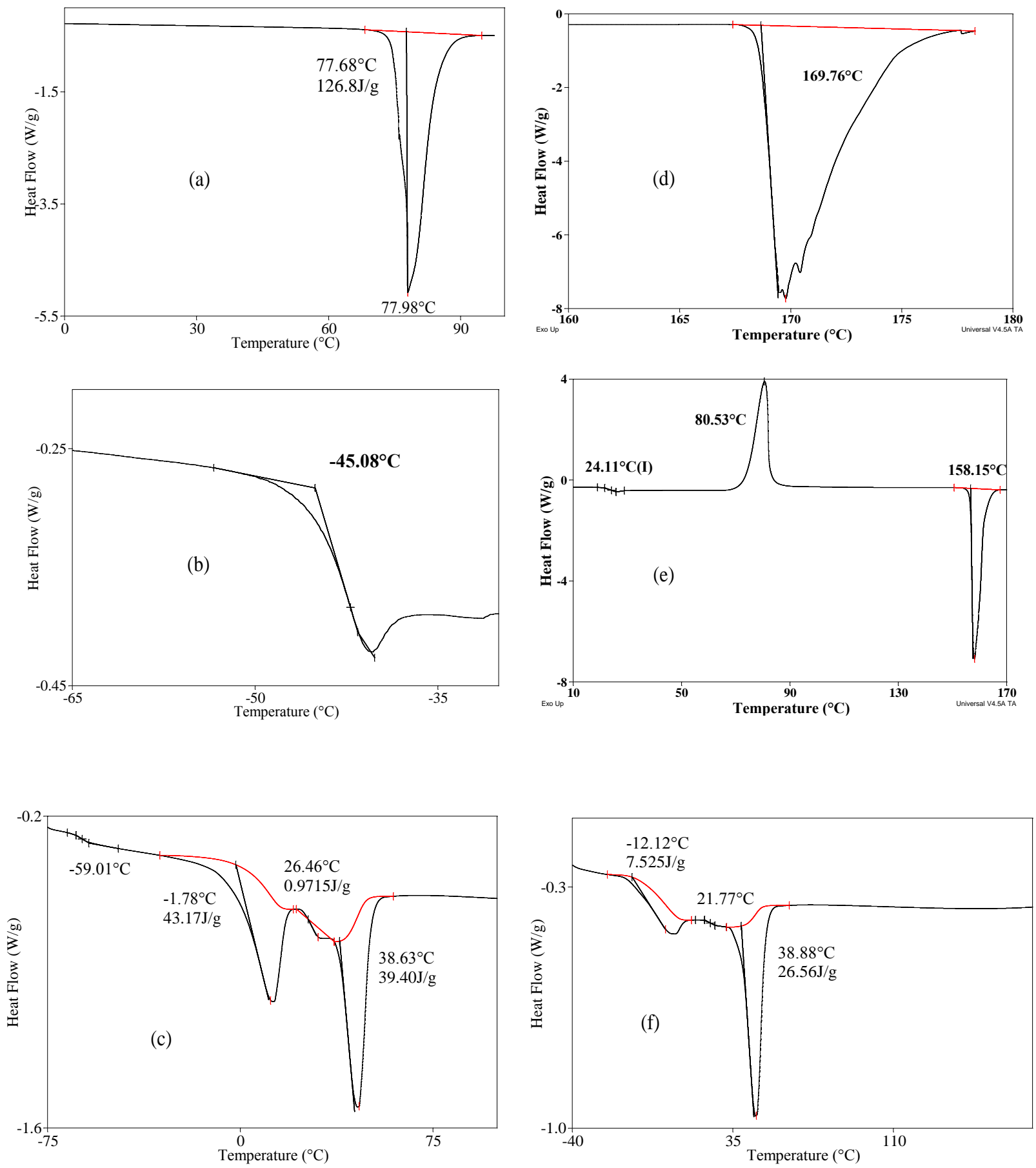

Figure 1 


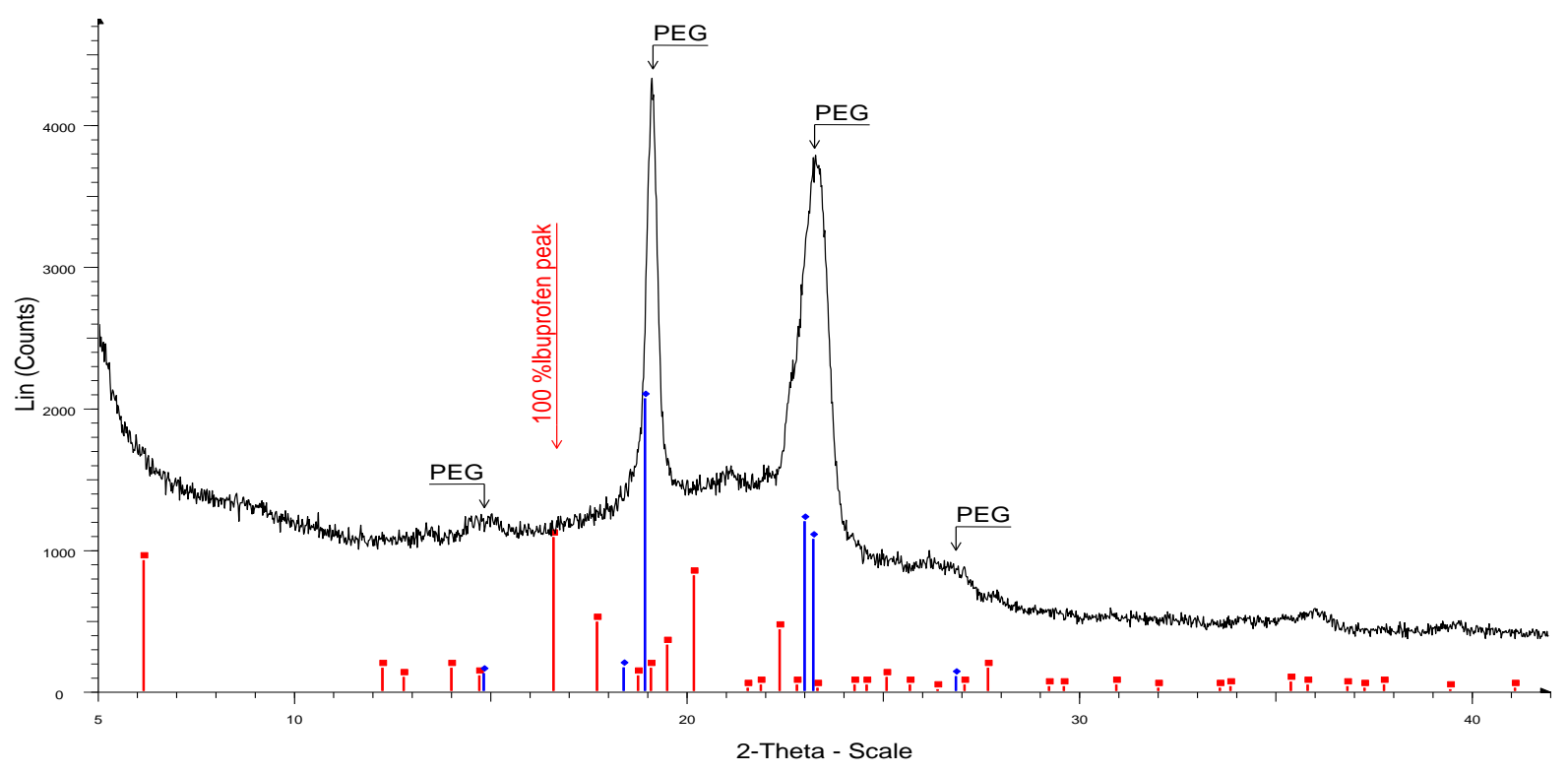

(a)

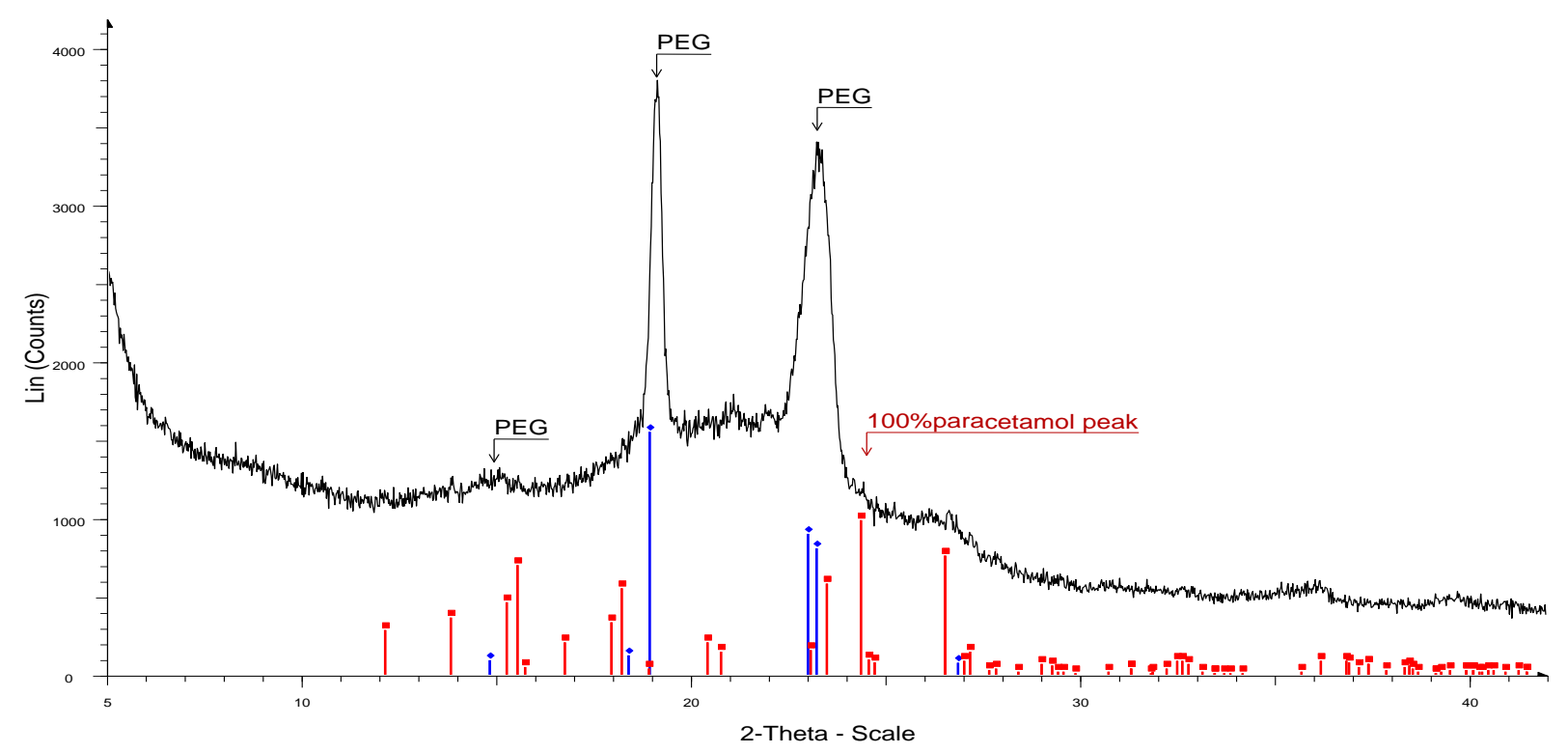

(b)

Figure 2 


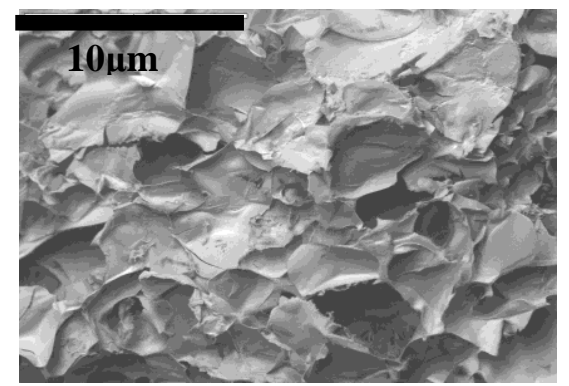

(a)

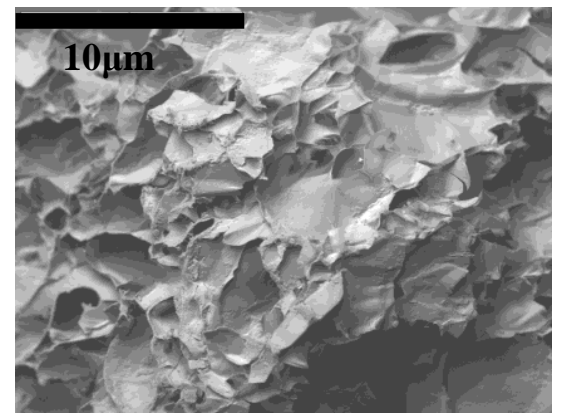

(c)

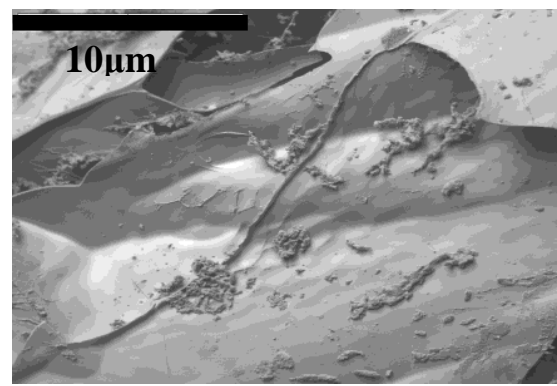

(b)

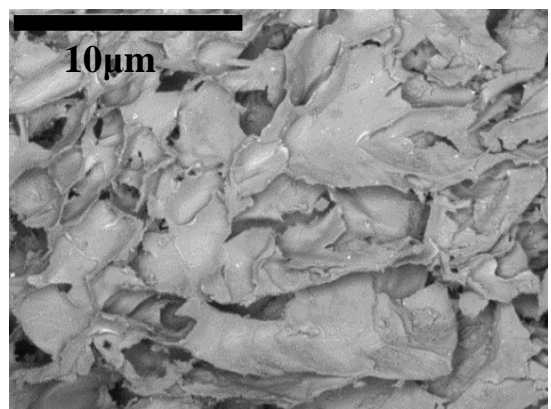

(d)

Figure 3 


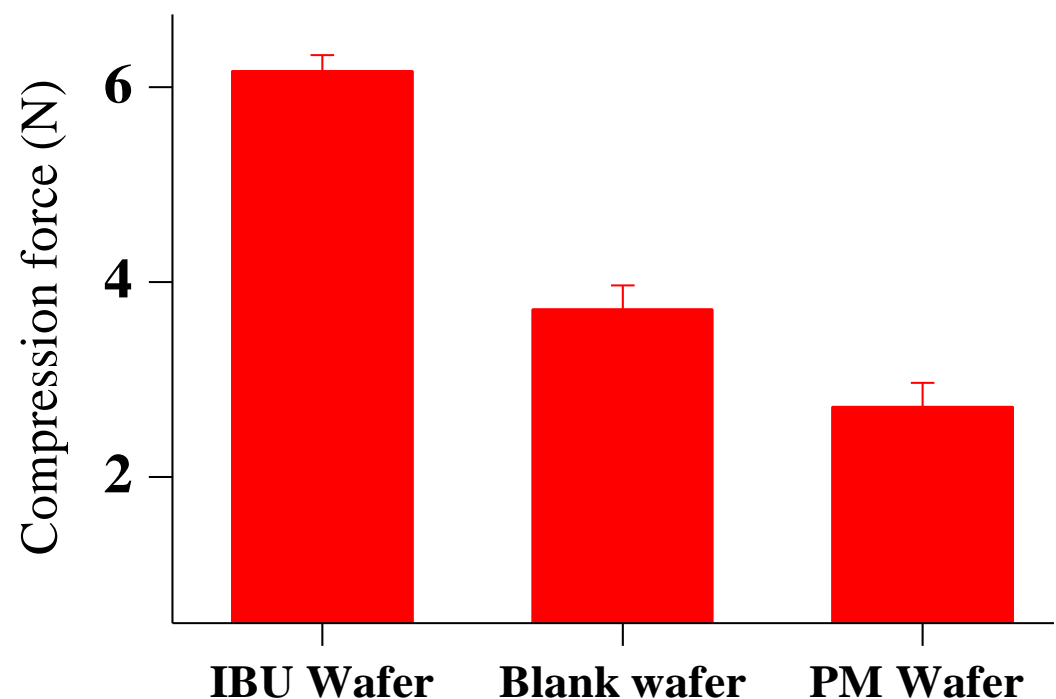

(a)

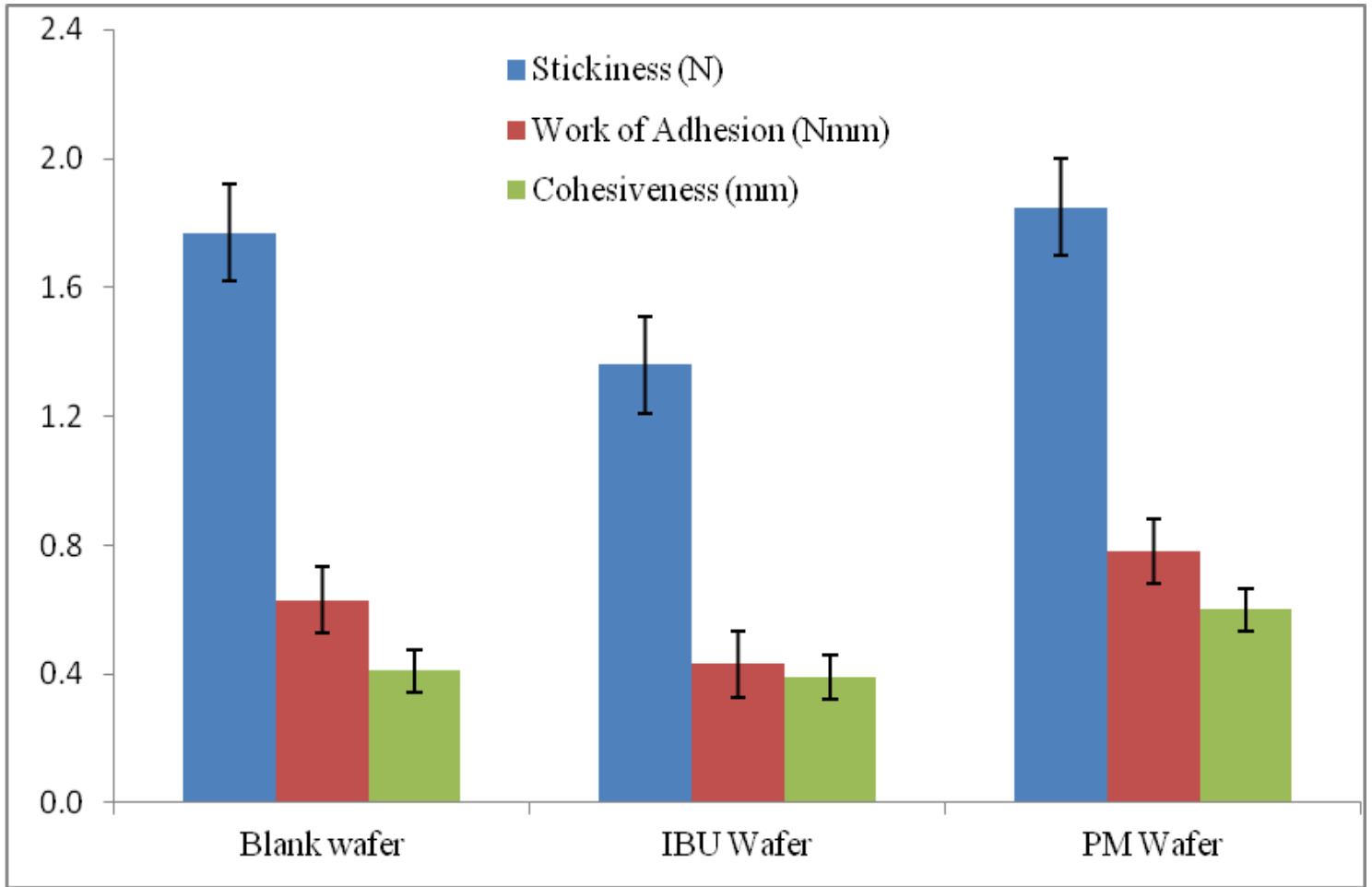

(b)

Figure 4

To be reproduced in colour on the Web (free of charge) and in black-and-white in print 


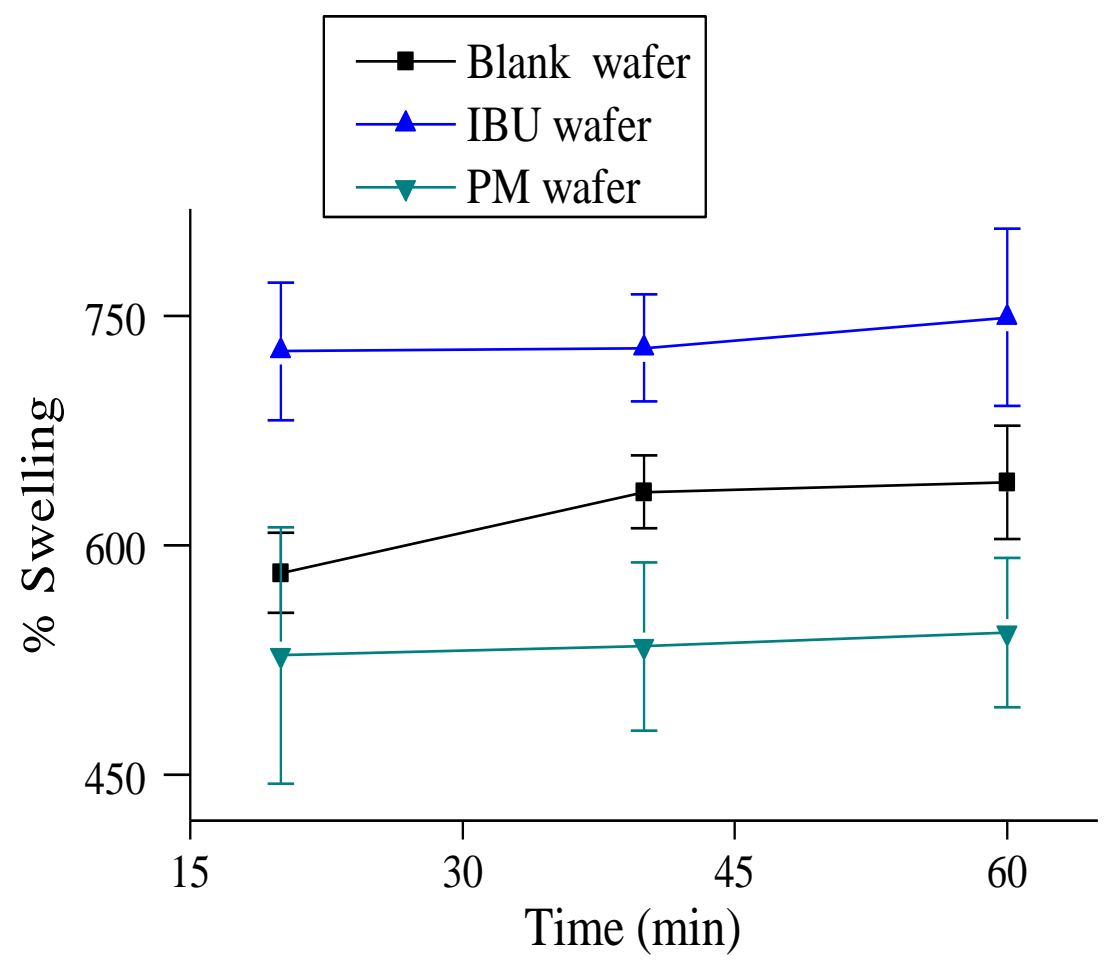

(a)

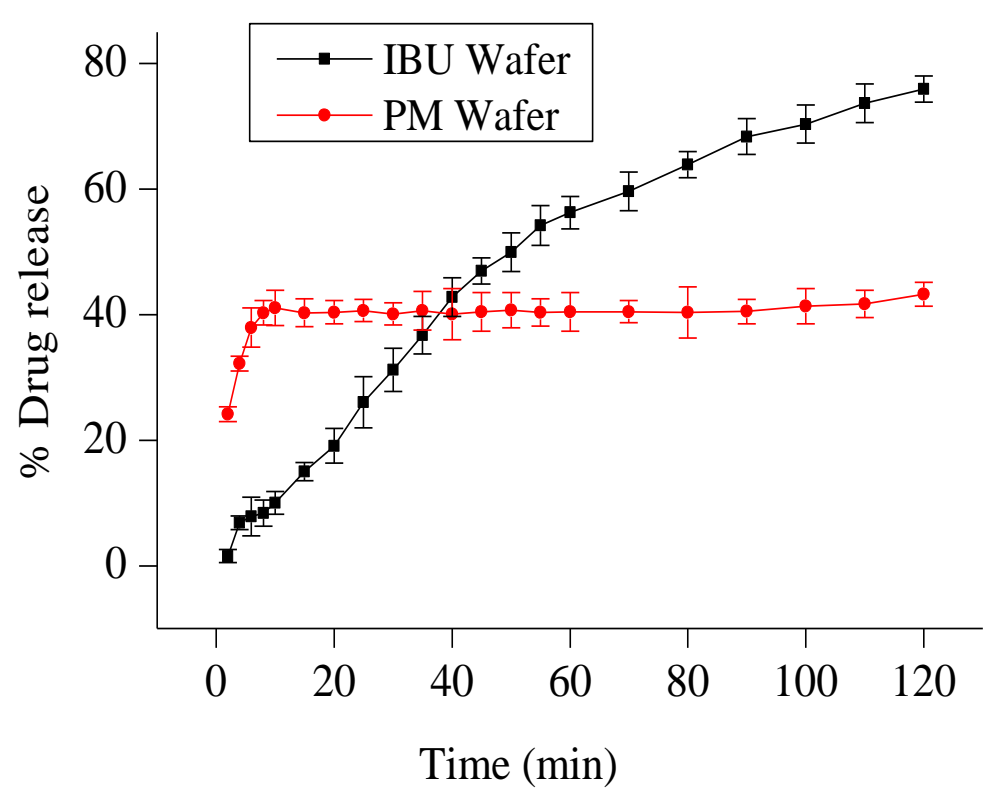

(b)

Figure 5

To be reproduced in colour on the Web (free of charge) and in black-and-white in print 